\title{
Predação de Diaspididae por larvas de Coccidophilus citricola em diferentes temperaturas
}

\author{
Predation of Diaspididae by Coccidophilus citricola larvae at different temperatures
}

\author{
Ricardo Adaime da Silva ${ }^{1}$ Marcos Doniseti Michelotto ${ }^{2}$ Antonio Carlos Busoli ${ }^{3}$ José Carlos Barbosa $^{4}$
}

\section{RESUMO}

O objetivo deste estudo foi avaliar o potencial de predação dos Diaspididae Aspidiotus nerii, Chrysomphalus aonidum, Selenaspidus articulatus e Parlatoria cinerea por larvas de Coccidophilus citricola Brèthes (Coleoptera: Coccinellidae) nas temperaturas de 19, 24 e $29 \pm 1^{\circ}$ C. S. articulatus foi a espécie predada em maior quantidade durante a fase larval de C. citricola, em todas as temperaturas. $O$ aumento da temperatura determinou redução do número de ninfas de C. aonidum predadas, ocorrendo o inverso para ninfas de $\mathbf{S}$. articulatus.

Palavras-chave: controle biológico, Aspidiotus nerii, Chrysomphalus aonidum, Selenaspidus articulatus, Parlatoria cinerea.

\section{ABSTRACT}

The objective of this study was to evaluate the predatory potential of Diaspididae Aspidiotus nerii, Chrysomphalus aonidum, Selenaspidus articulatus and Parlatoria cinerea by larvae of Coccidophilus citricola Brèthes (Coleoptera: Coccinellidae) at 19, 24 and $29 \pm 1^{\circ}$ C. S. articulatus was the species mostly preyed during the larval phase of $\boldsymbol{C}$. citricola, at all temperatures. The increment of temperature caused decrease on number of $C$. aonidum preyed nymphs, occurring the opposite for $\mathbf{S}$. articulatus.

Key words: biological control, Aspidiotus nerii, Chrysomphalus aonidum, Selenaspidus articulatus, Parlatoria cinerea.

\section{INTRODUÇÃO}

As "cochonilhas-de-carapaça" (Hemiptera: Diaspididae) podem causar danos severos às plantas, principalmente em decorrência da sucção contínua de seiva de folhas, frutos e ramos; além da introdução de toxinas que podem ocasionar queda de folhas ou depreciar a qualidade dos frutos produzidos (BARTRA, 1974). Nos ecossistemas citrícolas brasileiros, existe uma grande diversidade de diaspidídeos, que geralmente alcançam elevados índices populacionais (GALLO et al., 2002).

Coccidophilus citricola Brèthes (Coleoptera: Coccinellidae) é um dos predadores mais importantes das cochonilhas-de-carapaça e está amplamente distribuído na América do Sul (BOSQ, 1943; LIMA, 1948). Suas larvas são menores que $2 \mathrm{~mm}$ de comprimento quando completamente desenvolvidas (SILVA et al., 2002a). O adulto possui formato oval e alongado, coloração negra, os machos medem cerca de $1 \mathrm{~mm}$ e as fêmeas cerca de $1,25 \mathrm{~mm}$ de comprimento (GORDON, 1977). Larvas e adultos predam ativamente todos as fases de desenvolvimento da cochonilha Aspidiotus nerii Bouché, muito utilizada como presa na criação massal de coccinelídeos (SILVA et al., 2003).

A temperatura tem efeito significativo sobre o crescimento dos coccinelídeos (HAGEN, 1962; HODEK, 1967, 1973 e 1996; HONEK, 1996; IPERTI, 1999), podendo afetar a velocidade de procura de alimento (HONEK, 1996) e a taxa de consumo diário (HODEK, 1996).

Estudou-se o potencial de predação dos diaspidídeos A. nerii Bouché, Parlatoria cinerea Doane \& Hadden, Chrysomphalus aonidum (Linnaeus) e Selenaspidus articulatus (Morgan) por larvas de C. citricola, em diferentes temperaturas.

\footnotetext{
${ }^{1}$ Engenheiro Agrônomo, Doutor em Entomologia Agrícola, Pesquisador, Embrapa Amapá, Rodovia JK, Km 5, 68903-000, Macapá, AP. E-mail: ricardoadaime@ hotmail.com. Autor para correspondência.

${ }^{2}$ Engenheiro Agrônomo, Doutorando em Entomologia Agrícola, Faculdade de Ciências Agrárias e Veterinárias (FCAV), Universidade Estadual Paulista (UNESP), Via de Acesso Prof. Paulo Donato Castellane, s/n, 14884-900, Jaboticabal, SP.

${ }^{3}$ Engenheiro Agrônomo, Doutor em Entomologia, Professor Titular, Departamento de Fitossanidade, FCAV/UNESP.

${ }^{4}$ Engenheiro Agrônomo, Doutor em Estatística e Experimentação Agronômica, Professor Titular, Departamento de Ciências Exatas, FCAV/UNESP.
} 


\section{MATERIAL E MÉTODOS}

A pesquisa foi conduzida no Laboratório de Criação de Coccinelídeos e Diaspidídeos do Departamento de Fitossanidade da FCAV-UNESP, em Jaboticabal, SP.

As cochonilhas foram criadas sobre abóboras híbridas "Cabotiá" (Cucurbita moschata $\mathrm{x}$ Cucurbita maxima var. tetsukabuto), a $24 \pm 1{ }^{\circ} \mathrm{C}$, umidade relativa do ar de $70 \pm 10 \%$ e fotofase de 12 horas. Antes de serem utilizadas, as abóboras foram lavadas com água e sabão, com o auxílio de uma esponja, e posteriormente mergulhadas em solução de hipoclorito de sódio $(0,25 \%)$ por um minuto.

Uma linhagem uniparental de A. nerii, proveniente da Flórida, Estados Unidos, foi mantida em criação massal sobre abóboras dispostas em estantes de aço, seguindo os procedimentos descritos por ROSE (1990).

Para a criação das demais espécies de diaspidídeos, de reprodução sexuada, foram obtidos exemplares a partir de material vegetal oriundo de pomares de citros dos municípios de Jaboticabal e Taiúva, SP. As folhas foram coletadas e levadas ao laboratório, onde foram inspecionadas sob microscópio estereoscópio para selecionar as que continham adultos e, preferencialmente, ovos e ninfas móveis de $\boldsymbol{C}$. aonidum e $\boldsymbol{S}$. articulatus. Com auxílio de agulha histológica, foram removidas as outras espécies de cochonilhas e os ácaros presentes. As folhas selecionadas foram agrupadas e presas por alfinetes nos pedúnculos das abóboras, sendo estas acondicionadas em recipientes de plástico transparente $(30 \times 45 \times 30 \mathrm{~cm})$, com abertura na tampa revestida com "voil". Após sete dias, as folhas foram removidas e cada abóbora vistoriada para verificar se a quantidade de cochonilhas fixadas era suficiente ou se seria necessária nova colonização. As abóboras já colonizadas foram acondicionadas em bacias de plástico (50cm de diâmetro x $20 \mathrm{~cm}$ de altura) tampadas com vidro transparente de $0,5 \mathrm{~cm}$ de espessura, tendo duas aberturas laterais opostas, revestidas com "voil".

Foram coletados, com lâmina afiada, filetes da casca do tronco de plantas de citros contendo $\boldsymbol{P}$. cinerea, acondicionando o material em sacos de papel. A colonização das abóboras foi realizada de forma semelhante à descrita para as duas espécies anteriores, utilizando, no entanto, os filetes da casca.

Foram coletados cerca de 300 adultos de $\boldsymbol{C}$. citricola no tronco de laranjeiras colonizadas por Unaspis citri (Comstock) (Hemiptera: Diaspididae), em Jaboticabal, SP. Os coccinelídeos foram acondicionados em recipientes de plástico
$(18 \times 18 \times 25 \mathrm{~cm})$, cuja tampa continha uma abertura coberta com "voil". Em cada recipiente, foi colocada uma abóbora colonizada por $\boldsymbol{A}$. nerii e cerca de 100 coccinelídeos adultos. A cada 30 dias, os adultos foram transferidos para outras abóboras colonizadas pela cochonilha. Foi observado um período de 30 dias antes de descartar as abóboras, para possibilitar o desenvolvimento de larvas e a emergência de novos adultos. A criação foi realizada em sala climatizada, nas mesmas condições em que foram criadas as cochonilhas.

Para estudar o desenvolvimento dos imaturos do coccinelídeo e seu potencial de predação, foram utilizadas as "arenas de contenção de coccinelídeos” desenvolvidas por SILVA et al. (2002b), construídas a partir de frascos de filme fotográfico de coloração branca ( $3 \mathrm{~cm}$ de diâmetro), cortados a $2 \mathrm{~cm}$ de altura, com tampa vazada ( $2 \mathrm{~cm}$ de diâmetro), sob a qual foi colocado um pedaço de tecido de algodão. Todos os procedimentos de observação e avaliação das larvas foram realizados com o auxílio de um microscópio estereoscópio.

Foram selecionadas abóboras colonizadas com ninfas de $2^{\circ}$ estádio de cada espécie de cochonilha, que foram cortadas com lâmina afiada, em secções com 4 a $5 \mathrm{~cm}^{2}$. Para as espécies com reprodução sexuada, foram excluídas as carapaças dos machos, com o auxílio de agulhas histológicas. As porções laterais e inferior das secções de abóbora foram imersas em parafina líquida, com o auxílio de suporte metálico. Em cada arena foi disposta uma secção de abóbora já parafinada contendo cochonilhas de uma das espécies e um chumaço de algodão embebido em água destilada. Com um pincel de cerda única foi transferida uma larva de $\boldsymbol{C}$. citricola, recém-eclodida, para cada arena. Essas foram fechadas e acondicionadas em câmaras climatizadas a 19,24 e $29 \pm 1{ }^{\circ} \mathrm{C}$, umidade relativa do ar de $70 \pm 10 \%$ e fotofase de 12 horas. As larvas foram avaliadas duas vezes ao dia, observando se havia ocorrido ecdise e se era necessário repor o alimento. A cada mudança de estádio foi realizada a contagem das cochonilhas predadas pelas larvas, levantandose todas as carapaças, com o auxílio de agulha histológica. Foram consideradas predadas as cochonilhas cujo corpo havia sido parcial ou totalmente consumido.

O experimento foi realizado em delineamento inteiramente casualizado, com 10 repetições (larvas), em esquema fatorial $3 \times 4$, com 12 tratamentos correspondentes à combinação dos fatores temperatura $\left(19,24\right.$ e $\left.29 \pm 1^{\circ} \mathrm{C}\right)$ e espécie de presa $(\boldsymbol{A}$. nerii, $C$. aonidum, S. articulatus e $P$. cinerea). Os dados relativos à duração dos estádios e da fase larval 
e os respectivos números de cochonilhas predadas foram submetidos à análise de variância, sendo as médias comparadas pelo teste de Tukey a $5 \%$ de probabilidade de erro.

\section{RESULTADOS E DISCUSSÃO}

Desenvolvimento larval de $\boldsymbol{C}$. citricola. Houve efeito da interação entre os fatores temperatura e espécie de presa na duração do $1^{\circ}$ estádio de $\boldsymbol{C}$. citricola; do fator temperatura na duração do $2^{\circ}$ e $3^{\circ}$ estádios; dos fatores temperatura e espécie de presa na duração do 4o estádio e da fase larval (Tabela 1).

A duração do $1^{\circ}$ estádio, a $19^{\circ} \mathrm{C}$, foi maior em larvas alimentadas com $\boldsymbol{A}$. nerii e menor naquelas alimentadas com $\boldsymbol{P}$. cinerea . A $24^{\circ} \mathrm{C}$, a maior duração ocorreu em larvas alimentadas $\operatorname{com} \boldsymbol{A}$. nerii. A $29^{\circ} \mathrm{C}$, não houve influência das espécies de presas na duração do estádio. Todas as espécies de presas propiciaram maior duração do estádio a $19^{\circ} \mathrm{C}$ e menor a $29^{\circ} \mathrm{C}$, sendo que para larvas alimentadas com $S$. articulatus a duração do estádio a $24^{\circ} \mathrm{C}$ e $29^{\circ} \mathrm{C}$ não diferiu significativamente entre si.

$\mathrm{O} 2^{\circ}$ estádio foi maior a $19^{\circ} \mathrm{C}$ em relação às demais temperaturas. O $3^{\circ}$ e $4^{\circ}$ estádios e a fase larval foram maiores a $19^{\circ} \mathrm{C}$ e menores a $29^{\circ} \mathrm{C}$. A espécie $S$. articulatus apresentou a tendência de prolongar o $4^{\circ}$ estádio e a fase larval do predador (Tabela 1).

Número de ninfas predadas por larvas de C. citricola. Houve efeito dos fatores temperatura e espécie de presa no número de ninfas predadas no $1^{\circ}$ estádio de $\boldsymbol{C}$. citricola; da interação entre os fatores no $2^{\circ}$ e $3^{\circ}$ estádios e do fator espécie de presa no $4^{\circ}$ estádio (Tabela 2).

O maior número de ninfas predadas por larvas de $1^{\circ}$ estádio ocorreu a $29^{\circ} \mathrm{C}$ e o menor a $19^{\circ} \mathrm{C}$, sendo $\boldsymbol{S}$. articulatus a espécie predada em maior quantidade (Tabela 2).

No $2^{\circ}$ estádio larval, a $19^{\circ} \mathrm{C}$, o número de ninfas de $S$. articulatus predadas foi maior que o de $P$. cinerea. A $24^{\circ} \mathrm{C}$, não houve diferença no número de ninfas predadas entre as espécies. A $29^{\circ} \mathrm{C}$, o número de ninfas

Tabela 1.- Média ( \pm erro padrão) da duração, em dias, dos estádios larvais e da fase larval de $\boldsymbol{C}$. citricola, tendo como presas $\boldsymbol{A}$. $\boldsymbol{n e r i i}, \boldsymbol{P}$. cinerea, C. aonidum e $\boldsymbol{S}$. articulatus, nas temperaturas de 19,24 e $29^{\circ} \mathrm{C}$. UR de $70 \pm 10 \%$ e fotofase de 12 horas. Jaboticabal-SP, 2002 .

\begin{tabular}{|c|c|c|c|c|c|c|}
\hline \multirow{3}{*}{$\begin{array}{l}\text { Estádio } \\
\text { larval }\end{array}$} & \multirow{2}{*}{\multicolumn{5}{|c|}{ Espécies de }} & \multirow{3}{*}{ Média } \\
\hline & & & & & & \\
\hline & $\mathrm{T}\left({ }^{\circ} \mathrm{C}\right)$ & A. nerii & P. cinerea & C. aonidum & S. articulatus & \\
\hline \multirow{4}{*}{$1^{\mathrm{o}}$} & 19 & $12,06 \pm 0,70 \mathrm{aA}$ & $8,30 \pm 0,68 \mathrm{cA}$ & $9,94 \pm 0,69 \mathrm{bcA}$ & $11,36 \pm 0,56 a b A$ & $10,41 \pm 0,41$ \\
\hline & 24 & $7,65 \pm 0,63 \mathrm{aB}$ & $4,50 \pm 0,28 \mathrm{bB}$ & $4,95 \pm 0,52 \mathrm{bB}$ & $5,22 \pm 0,36 \mathrm{bB}$ & $5,58 \pm 0,31$ \\
\hline & 29 & $3,37 \pm 0,28 \mathrm{aC}$ & $2,77 \pm 0,21 \mathrm{aC}$ & $3,28 \pm 0,15 \mathrm{aC}$ & $3,80 \pm 0,19 \mathrm{aB}$ & $3,31 \pm 0,12$ \\
\hline & Média & $7,69 \pm 0,79$ & $5,19 \pm 0,50$ & $6,06 \pm 0,65$ & $6,79 \pm 0,72$ & $6,43 \pm 0,34$ \\
\hline \multirow{4}{*}{$2^{\underline{o}}$} & 19 & $9,33 \pm 1,36$ & $6,72 \pm 1,42$ & $6,31 \pm 0,44$ & $7,61 \pm 1,20$ & $7,49 \pm 0,56 \mathrm{~A}$ \\
\hline & 24 & $3,05 \pm 0,24$ & $2,69 \pm 0,40$ & $3,00 \pm 0,03$ & $2,83 \pm 0,14$ & $2,89 \pm 0,13 \mathrm{~B}$ \\
\hline & 29 & $1,88 \pm 0,17$ & $1,73 \pm 0,10$ & $1,75 \pm 0,15$ & $2,22 \pm 0,29$ & $1,89 \pm 0,10 \mathrm{~B}$ \\
\hline & Média & $4,75 \pm 0,82$ & $3,71 \pm 0,60$ & $3,69 \pm 0,43$ & $4,22 \pm 0,60$ & $4,09 \pm 0,30$ \\
\hline \multirow{4}{*}{$3^{\circ}$} & 19 & $7,35 \pm 0,63$ & $6,98 \pm 0,44$ & $7,10 \pm 0,68$ & $6,14 \pm 0,39$ & $6,89 \pm 0,25 \mathrm{~A}$ \\
\hline & 24 & $3,38 \pm 0,17$ & $3,01 \pm 0,23$ & $3,11 \pm 0,15$ & $3,72 \pm 0,27$ & $3,31 \pm 0,11 \mathrm{~B}$ \\
\hline & 29 & $2,06 \pm 0,20$ & $2,13 \pm 0,16$ & $2,49 \pm 0,11$ & $2,93 \pm 0,38$ & $2,40 \pm 0,15 \mathrm{C}$ \\
\hline & Média & $4,26 \pm 0,52$ & $4,04 \pm 0,46$ & $4,23 \pm 0,52$ & $4,26 \pm 0,34$ & $4,20 \pm 0,22$ \\
\hline \multirow{4}{*}{$4^{\circ}$} & 19 & $5,90 \pm 0,46$ & $6,89 \pm 0,58$ & $7,15 \pm 0,77$ & $8,29 \pm 1,86$ & $7,06 \pm 0,52 \mathrm{~A}$ \\
\hline & 24 & $3,54 \pm 0,18$ & $5,48 \pm 1,00$ & $2,95 \pm 0,29$ & $5,99 \pm 0,63$ & $4,49 \pm 0,38 \mathrm{~B}$ \\
\hline & 29 & $1,80 \pm 0,18$ & $2,18 \pm 0,28$ & $1,98 \pm 0,20$ & $4,47 \pm 0,48$ & $2,61 \pm 0,29 \mathrm{C}$ \\
\hline & Média & $3,75 \pm 0,41 \mathrm{~b}$ & $4,85 \pm 0,59 \mathrm{ab}$ & $4,03 \pm 0,58 \mathrm{~b}$ & $6,25 \pm 0,64 \mathrm{a}$ & $4,72 \pm 0,30$ \\
\hline \multirow{4}{*}{$\begin{array}{l}\text { Fase } \\
\text { larval }\end{array}$} & 19 & $35,40 \pm 2,90$ & $28,89 \pm 1,55$ & $30,75 \pm 1,52$ & $33,47 \pm 3,10$ & $32,13 \pm 1,16 \mathrm{~A}$ \\
\hline & 24 & $17,16 \pm 0,95$ & $15,70 \pm 1,08$ & $14,01 \pm 0,62$ & $17,77 \pm 0,61$ & $16,16 \pm 0,48 \mathrm{~B}$ \\
\hline & 29 & $9,30 \pm 0,53$ & $8,62 \pm 0,31$ & $9,65 \pm 0,54$ & $13,42 \pm 0,61$ & $10,25 \pm 0,48 \mathrm{C}$ \\
\hline & Média & $20,62 \pm 2,64 \mathrm{ab}$ & $17,74 \pm 1,93 b$ & $18,14 \pm 2,13 b$ & $21,55 \pm 1,97 \mathrm{a}$ & $19,51 \pm 1,06$ \\
\hline
\end{tabular}

${ }^{1}$ Médias não seguidas de mesma letra minúscula na linha e maiúscula na coluna diferem significativamente entre si pelo teste de Tukey em nível de $5 \%$ de probabilidade de erro.

Ciência Rural, v.34, n.5, set-out, 2004. 
Tabela 2.- Média ( \pm erro padrão) do número de ninfas de $\boldsymbol{A}$. nerii, $\boldsymbol{P}$. cinerea, $\boldsymbol{C}$. aonidum e $\boldsymbol{S}$. articulatus predadas durante os estádios larvais e a fase larval de $\boldsymbol{C}$. citricola, nas temperaturas de 19,24 e $29^{\circ} \mathrm{C}$. UR de $70 \pm 10 \%$ e fotofase de 12 horas. Jaboticabal-SP, 2002 .

\begin{tabular}{|c|c|c|c|c|c|c|}
\hline \multirow{2}{*}{$\begin{array}{l}\text { Estádio } \\
\text { larval }\end{array}$} & \multirow{2}{*}{$\mathrm{T}\left({ }^{\circ} \mathrm{C}\right)$} & \multicolumn{4}{|c|}{ Espécies de presas $^{1}$} & \multirow{2}{*}{ Média } \\
\hline & & A. nerii & P. cinerea & C. aonidum & S. articulatus & \\
\hline \multirow{4}{*}{$1^{\circ}$} & 19 & $2,86 \pm 0,26$ & $4,57 \pm 0,61$ & $3,75 \pm 0,62$ & $8,43 \pm 1,23$ & $4,90 \pm 0,53 \mathrm{~B}$ \\
\hline & 24 & $3,22 \pm 0,28$ & $3,89 \pm 0,42$ & $4,63 \pm 0,73$ & $9,88 \pm 0,91$ & $5,41 \pm 0,54 \mathrm{AB}$ \\
\hline & 29 & $5,71 \pm 0,57$ & $4,56 \pm 0,71$ & $5,13 \pm 0,44$ & $10,88 \pm 1,09$ & $6,57 \pm 0,58 \mathrm{~A}$ \\
\hline & Média & $3,93 \pm 0,33 \mathrm{~b}$ & $4,34 \pm 0,33 \mathrm{~b}$ & $4,50 \pm 0,35 \mathrm{~b}$ & $9,73 \pm 0,65 \mathrm{a}$ & $5,63 \pm 0,32$ \\
\hline \multirow{4}{*}{$2^{-}$} & 19 & $8,17 \pm 1,80 \mathrm{abA}$ & $5,57 \pm 0,84 \mathrm{bA}$ & $8,57 \pm 0,48 \mathrm{abA}$ & $12,00 \pm 1,93 \mathrm{aB}$ & $8,58 \pm 0,77$ \\
\hline & 24 & $7,63 \pm 1,10 \mathrm{aA}$ & $6,11 \pm 0,45 \mathrm{aA}$ & $6,13 \pm 0,55 \mathrm{aA}$ & $11,25 \pm 1,46 \mathrm{aB}$ & $7,78 \pm 0,59$ \\
\hline & 29 & $7,17 \pm 0,91 \mathrm{bA}$ & $5,25 \pm 0,56 \mathrm{bA}$ & $5,71 \pm 0,64 \mathrm{bA}$ & $22,13 \pm 3,07 \mathrm{aA}$ & $10,07 \pm 1,61$ \\
\hline & Média & $7,66 \pm 0,71$ & $5,64 \pm 0,34$ & $6,80 \pm 0,41$ & $15,13 \pm 1,69$ & $8,81 \pm 0,62$ \\
\hline \multirow{4}{*}{$3^{\mathrm{o}}$} & 19 & $13,00 \pm 0,89 \mathrm{aA}$ & $12,29 \pm 1,92 \mathrm{aA}$ & $9,43 \pm 0,37 \mathrm{aA}$ & $14,50 \pm 1,26 \mathrm{aB}$ & $12,31 \pm 0,72$ \\
\hline & 24 & $11,63 \pm 1,98 \mathrm{bA}$ & $9,44 \pm 1,00 \mathrm{bA}$ & $11,63 \pm 1,85 \mathrm{bA}$ & $24,13 \pm 3,95 \mathrm{aA}$ & $14,21 \pm 1,52$ \\
\hline & 29 & $11,00 \pm 1,75 \mathrm{bA}$ & $13,71 \pm 2,06 \mathrm{bA}$ & $9,20 \pm 0,58 \mathrm{bA}$ & $28,63 \pm 3,12 \mathrm{aA}$ & $15,64 \pm 1,96$ \\
\hline & Média & $11,88 \pm 1,00$ & $11,81 \pm 0,98$ & $10,09 \pm 0,78$ & $22,42 \pm 2,16$ & $14,05 \pm 0,89$ \\
\hline \multirow{4}{*}{$4^{\circ}$} & 19 & $18,80 \pm 1,74$ & $19,14 \pm 1,87$ & $25,86 \pm 0,96$ & $36,67 \pm 4,28$ & $25,12 \pm 1,85$ \\
\hline & 24 & $23,14 \pm 4,08$ & $31,38 \pm 1,58$ & $18,75 \pm 2,49$ & $39,50 \pm 5,64$ & $28,20 \pm 2,31$ \\
\hline & 29 & $22,67 \pm 4,20$ & $29,33 \pm 4,01$ & $13,80 \pm 2,08$ & $39,88 \pm 3,49$ & $26,41 \pm 2,61$ \\
\hline & Média & $21,54 \pm 2,10 b$ & $26,62 \pm 1,82 b$ & $19,47 \pm 1,55 b$ & $38,68 \pm 2,57 \mathrm{a}$ & $26,58 \pm 1,32$ \\
\hline \multirow{4}{*}{$\begin{array}{l}\text { Fase } \\
\text { larval }\end{array}$} & 19 & $43,60 \pm 3,39 \mathrm{bA}$ & $41,57 \pm 2,15 \mathrm{bA}$ & $47,86 \pm 1,20 \mathrm{bA}$ & $71,33 \pm 6,82 \mathrm{aB}$ & $51,09 \pm 2,98$ \\
\hline & 24 & $46,14 \pm 3,88 \mathrm{bA}$ & $51,25 \pm 2,47 \mathrm{bA}$ & $41,13 \pm 2,64 \mathrm{bAB}$ & $84,75 \pm 4,96 \mathrm{aB}$ & $55,82 \pm 3,59$ \\
\hline & 29 & $47,00 \pm 3,49 \mathrm{bcA}$ & $52,50 \pm 6,01 \mathrm{bA}$ & $33,00 \pm 1,58 \mathrm{cB}$ & $101,50 \pm 4,96 \mathrm{aA}$ & $58,50 \pm 5,98$ \\
\hline & Média & $45,58 \pm 2,03$ & $48,44 \pm 2,24$ & $40,66 \pm 1,74$ & $85,86 \pm 3,99$ & $55,14 \pm 2,50$ \\
\hline
\end{tabular}

${ }^{1}$ Médias não seguidas de mesma letra minúscula na linha e maiúscula na coluna diferem significativamente entre si pelo teste de Tukey em nível de $5 \%$ de probabilidade de erro.

predadas foi maior em $\boldsymbol{S}$. articulatus. A elevação da temperatura de $24^{\circ} \mathrm{C}$ para $29^{\circ} \mathrm{C}$ propiciou aumento no número de ninfas de $\boldsymbol{S}$. articulatus predadas (Tabela 2).

Durante o $3^{\circ}$ estádio, as larvas criadas a $19^{\circ} \mathrm{C}$ não apresentaram diferença significativa quanto ao número de ninfas predadas entre as espécies. A $24^{\circ} \mathrm{C}$ e $29^{\circ} \mathrm{C}$, S. articulatus foi a espécies mais predada. Ocorreu aumento no número de ninfas de $S$. articulatus predadas em temperaturas acima de $19^{\circ} \mathrm{C}$ (Tabela 2).

A espécie de cochonilha predada em maior quantidade durante o $4^{\circ}$ estádio larval foi $S$. articulatus (Tabela 2).

Considerando toda a fase larval de $\boldsymbol{C}$. citricola, houve efeito da interação entre os fatores temperatura e espécie de presa no número de ninfas predadas (Tabela 2). Em todas as temperaturas, a espécie predada em maior quantidade foi S. articulatus. Isso pode ser explicado pelas características morfológicas da cochonilha, que apresenta uma carapaça muito tênue, facilmente rompida pelas mandíbulas do predador, sendo também pouco espessa em relação às demais espécies de diaspidídeos estudadas. Dessa forma, o corpo da cochonilha contém pouca quantidade de líquido e, conseqüentemente, nutrientes em seu corpo. Como relatado por SILVA et al. (2003), no processo de alimentação, após romper a carapaça da cochonilha, a larva aspira todo o conteúdo líquido do corpo da mesma. Portanto, para obter os nutrientes necessários ao seu desenvolvimento, as larvas necessitam de uma maior quantidade de ninfas de $S$. articulatus em relação às demais espécies. De forma semelhante, RICCI (1986) observou que o comportamento alimentar de $\boldsymbol{C}$. citricola pode variar de acordo com a consistência da presa, ocorrendo a predação geralmente nos setores menos resistentes da carapaça. Como exemplo, o autor refere que as larvas atacam a zona caudal da carapaça dos machos de Pinnaspis aspidistrae (Signoret) e as zonas lateral e caudal da carapaça das fêmeas de Insulaspis gloverii (Packard).

Larvas alimentadas com $\boldsymbol{A}$. nerii e P. cinerea não sofreram influência da temperatura no número total de ninfas predadas durante a fase larval (Tabela 2). Para as larvas alimentadas com $\boldsymbol{C}$. aonidum, a elevação da temperatura proporcionou menor consumo de ninfas, ocorrendo o oposto para $S$. articulatus (Tabela 2). Os resultados obtidos estão de acordo com HODEK (1996), 
que em extensa revisão a respeito dos hábitos alimentares dos coccinelídeos, considera o consumo total durante a fase larval desses insetos praticamente estável em diferentes temperaturas constantes, ocorrendo pouca variação. Geralmente, o que varia é somente a taxa de consumo diário, pois a elevação da temperatura provoca redução da duração da fase larval (HODEK, 1967 e 1996).

No caso das larvas alimentadas com $\boldsymbol{S}$. articulatus, o fato de ter havido maior quantidade de ninfas predadas a $29^{\circ} \mathrm{C}$ (Tabela 2 ) possivelmente seja em decorrência da maior necessidade de consumo de água nas temperaturas mais elevadas, como verificado por GYENGE et al. (1998) em larvas de Eriopis connexa (Germar) (Coleoptera: Coccinellidae). Segundo WIGGLESWORTH(1974), a água tem papel fundamental na relação dos insetos com as altas temperaturas do meio, pois nessas condições os mesmos podem morrer pela temperatura propriamente dita ou pela dessecação decorrente da evaporação.

Ocasionalmente, larvas e adultos de coccinelídeos não conseguem predar totalmente uma cochonilha. Mesmo assim, tais pragas podem ser consideradas predadas, pois tornam-se mais vulneráveis ao ataque de outros insetos e à infecção por microrganismos patogênicos (De BORTOLI et al., 2001).

\section{CONCLUSÃO}

S. articulatus foi a espécie predada em maior quantidade durante a fase larval de $\boldsymbol{C}$. citricola, em todas as temperaturas. O aumento da temperatura determinou redução do número de ninfas de $\boldsymbol{C}$. aonidum predadas, ocorrendo comportamento inverso para ninfas de $S$. articulatus.

\section{AGRADECIMENTO}

Os autores agradecem à FAPESP (Fundação de Amparo à Pesquisa do Estado de São Paulo) pela bolsa de Doutorado concedida ao primeiro autor e pelo suporte financeiro.

\section{REFERÊNCIAS BIBLIOGRÁFICAS}

BARTRA, P.C.E. Biologia de Selenaspidus articulatus Morgan y sus principales controladores biológicos. Revista Peruana de Entomologia, Lima, v.17, n.1, p.60-68, 1974.

BOSQ, J.M. Coccinélideos útiles para la fruticultura tucumana. Revista Sociedade Entomologica Argentina, Buenos Aires, n.11, p.461-70, 1943 .

De BORTOLI, S.A. et al. Biologia de Pentilia egena Mulsant (Coleoptera: Coccinellidae) e predação sobre Chrysomphalus ficus Ashmead (Homoptera: Diaspididae). Boletín de Sanidad Vegetal Plagas, Madrid, v.27, p.337-343, 2001.

GALLO, D. et al. Entomologia agrícola. Piracicaba : FEALQ, 2002. 920p.
GORDON, R.D. Classification and phylogeny of the new world Sticholotidinae (Coccinellidae). Coleopterists Bulletin, Chicago, v 31, n.3, p.185-228, 1977.

GYENGE, J.E.; EDELSTEIN, J.D.; SALTO, C.E. Efectos de la temperatura y la dieta en la biología de Eriopis connexa (Germar) (Coleoptera: Coccinellidae). Anais da Sociedade Entomológica do Brasil, Londrina, v.27, n.3, p.345-356, 1998.

HAGEN, K.S. Biology and ecology of predaceous Coccinellidae. Annual Review of Entomology, Palo Alto, v.7, p.289-326, 1962.

HODEK, I. Bionomics and ecology of predaceous Coccinellidae Annual Review of Entomology, Palo Alto, v.12, p.79-104, 1967.

HODEK, I. Biology of Coccinellidae. Prague : Academy of Sciences, 1973. 260p.

HODEK, I. Food relationships. In: HODEK, I.; HONEK, A. Ecology of Coccinellidae. Dordrecht : Kluwer Academic, 1996. p.143-238.

HONEK, A. Life history and development. In: HODEK, I.; HONEK, A. Ecology of Coccinellidae. Dordrecht : Kluwer Academic, 1996. Chapter 4, p.61-93.

IPERTI, G. Biodiversity of predaceous coccinellidae in relation to bioindication and economic importance. Agriculture, Ecosystems and Environment, Amsterdam, v.74, p.323-342, 1999.

LIMA, A.M. da C. Entomófagos sulamericanos (parasitos e predadores) de insetos nocivos a agricultura. Boletim Sociedade Brasileira de Agronomia, Rio de Janeiro, v.11, n.1, p.1-32, 1948.

RICCI, J.G. Breve descripción, hábitos de alimentación y enemigos naturales de tres coccinélidos predadores de diaspídidos en cítricos de Tucumán (Republica Argentina) (1). CIRPON - Revista de Investigación, San Miguel de Tucumán, v.4, n.1-4, p.7-25, 1986.

ROSE, M. Rearing and mass rearing. In: ROSEN, D. Armored scale insects their biology, natural enemies and control. Jerusalém : Elseiver, 1990. p.357-365.

SILVA, R.A. et al. Descrição dos estágios imaturos de Coccidophilus citricola Brèthes, 1905 (Coleoptera: Coccinellidae). In: CONGRESSO BRASILEIRO DE ENTOMOlOGiA, 19., 2002, Manaus, AM. Resumos... Manaus : INPA, 2002a. p.237.

SILVA, R.A.; CHAGAS-FILHO, N.R.; BUSOLI, A.C. Técnica alternativa de contenção de coccinelídeos predadores de diaspidídeos para estudos bioecológicos. In: REUNIÃO ANUAL DA SOCIEDADE BRASILEIRAPARAO PROGRESSODACIÊNCIA, 54., 2002, Goiânia, GO. Resumos... Goiânia: SBPC/UFG, 2002b. 1CD.

SILVA, R.A. et al. Desenvolvimento e comportamento de predação de Coccidophilus citricola Brèthes, 1905 (Coleoptera: Coccinellidae) sobre Aspidiotus nerii Bouché, 1833 (Hemiptera: Diaspididae). Boletín de Sanidad Vegetal Plagas, Madrid, v.29, n.1, p.9-15, 2003.

WIGGLESWORTH, V.B. Nutrición y metabolismo. In: WIGGLESWORTH, V.B. Fisiología de los insectos. Zaragoza: Acribia, 1974. p.74-90. 